\title{
In vivo modification of tyrosine residues in recombinant mussel adhesive protein by tyrosinase co-expression in Escherichia coli
}

\author{
Yoo Seong Choi ${ }^{1}$, Yun Jung Yang ${ }^{2}$, Byeongseon Yang ${ }^{2}$ and Hyung Joon Cha ${ }^{2,3^{*}}$
}

\begin{abstract}
Background: In nature, mussel adhesive proteins (MAPs) show remarkable adhesive properties, biocompatibility, and biodegradability. Thus, they have been considered promising adhesive biomaterials for various biomedical and industrial applications. However, limited production of natural MAPs has hampered their practical applications. Recombinant production in bacterial cells could be one alternative to obtain useable amounts of MAPs, although additional post-translational modification of tyrosine residues into 3,4-dihydroxyphenyl-alanine (Dopa) and Dopaquinone is required. The superior properties of MAPs are mainly attributed to the introduction of quinone-derived intermolecular cross-links. To solve this problem, we utilized a co-expression strategy of recombinant MAP and tyrosinase in Escherichia coli to successfully modify tyrosine residues in vivo.

Results: A recombinant hybrid MAP, fp-151, was used as a target for in vivo modification, and a dual vector system of pET and pACYC-Duet provided co-expression of fp-151 and tyrosinase. As a result, fp-151 was over-expressed and mainly obtained from the soluble fraction in the co-expression system. Without tyrosinase co-expression, fp-151 was over-expressed in an insoluble form in inclusion bodies. The modification of tyrosine residues in the soluble-expressed fp-151 was clearly observed from nitroblue tetrazolium staining and liquid-chromatography-mass/mass spectrometry analyses. The purified, in vivo modified, fp-151 from the co-expression system showed approximately 4-fold higher bulk-scale adhesive strength compared to in vitro tyrosinase-treated fp-151.
\end{abstract}

Conclusion: Here, we reported a co-expression system to obtain in vivo modified MAP; additional in vitro tyrosinase modification was not needed to obtain adhesive properties and the in vivo modified MAP showed superior adhesive strength compared to in vitro modified protein. It is expected that this co-expression strategy will accelerate the use of functional MAPs in practical applications and can be successfully applied to prepare other Dopa/Dopaquinone-based biomaterials.

Keywords: Mussel adhesive protein, Dopa, Dopaquinone, In vivo modification, Tyrosinase, Co-expression, Escherichia coli

\section{Background}

To live in tidal aqueous environments and to protect themselves from predators, marine sessile organisms, such as mussels and tubeworms, attach themselves to hard substratum using protein-based adhesives [1,2]. Marinederived protein bioadhesives are considered promising

\footnotetext{
*Correspondence: hjcha@postech.ac.kr

${ }^{2}$ Department of Chemical Engineering, Pohang University of Science and Technology, Pohang 790-784, Korea

${ }^{3}$ Ocean Science and Technology Institute, Pohang University of Science and Technology, Pohang 790-784, Korea

Full list of author information is available at the end of the article
}

biomaterials in medical, environmental, and industrial applications because of their versatile adhesive properties, such as strong and flexible adhesion, durability, biodegradability, and biocompatibility [2,3]. Remarkably, these properties are mainly attributable to the introduction of quinone-derived intermolecular cross-links among individual adhesive proteins (quinone tanning) [4]. Hydroxylation of tyrosine residues leads to the formation of 3,4-dihydroxyphenyl-alanine (Dopa), which plays an important role in the adhesion and cross-linking of a catecholic precursor. Dopa enables adhesive proteins to cross-link via aryl

\section{Biomed Central}


coupling, thiol addition, coordination of multiple ligands by $\mathrm{Fe}^{3+}$, and chemisorption by chelation of surface metal oxides [5]. High levels of Dopa content are observed in mussel adhesive proteins (MAPs; 3 30 mol\%) [6] and tubeworm cement proteins (7 10 mol\%) [7].

MAPs have been extensively studied over the last 30 years as a characteristic example of Dopa-based biological adhesion. Six distinct adhesive proteins of type 1 (fp-1) through type 6 (fp-6) have been identified from adhesive plaque, and the proteins can be extracted by acid-based chemical extraction processes $[4,6]$. However, the extracted amount is not practically applicable for bioadhesives as 10,000 mussels are required to obtain $\sim 1 \mathrm{~g}$ of the most extractable MAP $\mathrm{fp}-1$, and at least $100 \mathrm{mg}$ of protein is needed for small, conventional adhesive tests, such as tensile or shear strength analysis, for obtaining mechanical properties of adhesive biomaterials $[2,8]$. Thus, recombinant MAP expression systems have been applied to overcome the low production yield of natural extraction, but posttranslational modifications, such as Dopa and Dopaquinone conversion, do not always occur naturally in recombinant systems [2,6]. Especially, recombinant MAPs from high yield bacterial expression systems require additional modifications to become functional. The modification of tyrosine residues can be accomplished in vitro with mushroom tyrosinase [9]. Some studies have shown that the levels of Dopa are related to the adhesive properties [10-12]. MAPs tend to easily aggregate in solution due to their high levels of aromatic and basic amino acids [13-15]. In addition, tyrosinase is known to most effectively oxidize free tyrosines rather than those bound within large protein and tyrosine residues within monomeric protein rather than protein aggregate $[16,17]$. Thus, the in vitro modification yield is relatively low due to limited access of the tyrosine residues within MAP [12]. To improve the modification efficiency, the modification with tyrosinase needs to be conducted as soon as the protein is expressed in vivo. Thus, an in vivo modification system for tyrosine residues, such as natural MAP production in mussel, is required to prepare recombinant MAPs in bacterial expression systems.

In the present work, for the first time, we co-expressed functional tyrosinase with recombinant MAP in Escherichia coli to efficiently modify tyrosine residues in vivo. The recombinant hybrid MAP fp-151 [18], which is composed of six fp-1 decapeptide repeats at both termini of fp-5, was used as a model. Previously, high production yield ( 1 g-purified protein per 1 L-pilot-scale fed-batch culture) and improved solubility ( $>300 \mathrm{~g} / \mathrm{L})$ of $\mathrm{fp}-151$ showed strong potential as a practical bioadhesive [18].

\section{Results and discussion}

\section{Co-expression of $\mathrm{fp}-151$ and tyrosinase}

Previously, we successfully over-expressed fp-151 ( 40\% of total protein) using a $\mathrm{pET}$ vector system with strong
T7 promoter and pBR322 origin (right vector map, Figure 1A) [18]. Because the pET vector can be used in combination with a vector system with $\mathrm{p} 15 \mathrm{~A}$ replicon [19] and functional expression of tyrosinase from Streptomyces antibioticus was reported in E. coli under the control of T7 promoter [20], recombinant pACYC-Tyr438 plasmid (left vector map, Figure 1A) was prepared from pACYCDuet-1, which carries the p15A origin. For functional expression of recombinant tyrosinase, ORF 438 , which is essential for copper incorporation into the active site of tyrosinase [21], was also co-expressed under the T7 promoter. Then, both recombinant pETfp151 and pACYC-Tyr-438 plasmids were introduced into $E$. coli BL21(DE3) to obtain in vivo tyrosinemodified fp-151 (Figure 1B).

Recombinant fp-151 was successfully co-expressed with tyrosinase and ORF 438 in the dual vector system (Figure 2A). We found that its expression level was similar to that of $\mathrm{fp}-151$ from $\mathrm{pET}$-fp151 alone. In addition, the growth of E. coli in the co-expression system displayed a similar trend to that of $E$. coli in the sole fp-151expression system (data not shown). Thus, the co-expression system does not seem to harm the growth of the host. Interestingly, the co-expressed fp151 (cofp-151) was observed in both the soluble (mainly) and insoluble fractions, whereas the fp-151 alone was mostly expressed in the form of insoluble inclusion bodies. The soluble fp-151 in SDS-PAGE was separated at a higher molecular weight than its calculated molecular mass $(\sim 24 \mathrm{kDa})$, which was also observed in previous reports and other recombinant MAPs $[18,22,23]$. Tyrosinase $(\sim 32 \mathrm{kDa})$ in the dual vector system was also co-expressed both as soluble and insoluble forms (Figure 2A), although the expression level was significantly decreased compared to that from E. coli BL21(DE3) bearing the pACYC-Tyr-438 plasmid only; tyrosinase from the pACYC-Tyr-438 alone was obtained in insoluble inclusion bodies at $37^{\circ} \mathrm{C}$ culture $(\sim 45 \%$ of total protein) and as a soluble protein at $20^{\circ} \mathrm{C}$ culture ( $15 \%$ of total protein) after IPTG induction (data not shown). ORF 438 ( $15 \mathrm{kDa})$ was only expressed in a soluble form in the dual vector system (Figure $2 \mathrm{~A}$ ). We surmised that soluble expression of recombinant fp-151 in the dual vector system might be from its modification by functional tyrosinase co-expression.

\section{Purification of cofp-151}

Affinity chromatography was conducted to purify the cofp-151 from the soluble fraction. Affinity purification was not successful under native conditions because the cofp-151 bound poorly to the Ni-NTA resin (data not shown). When gel filtration chromatography was applied, a group of the largest molecules was separated as a high resolution fraction from the remainder of the 

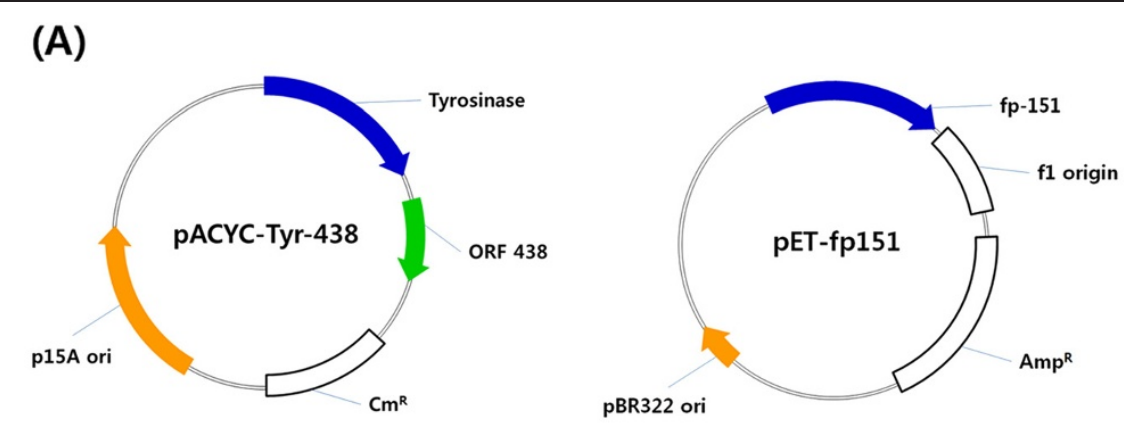

(B)

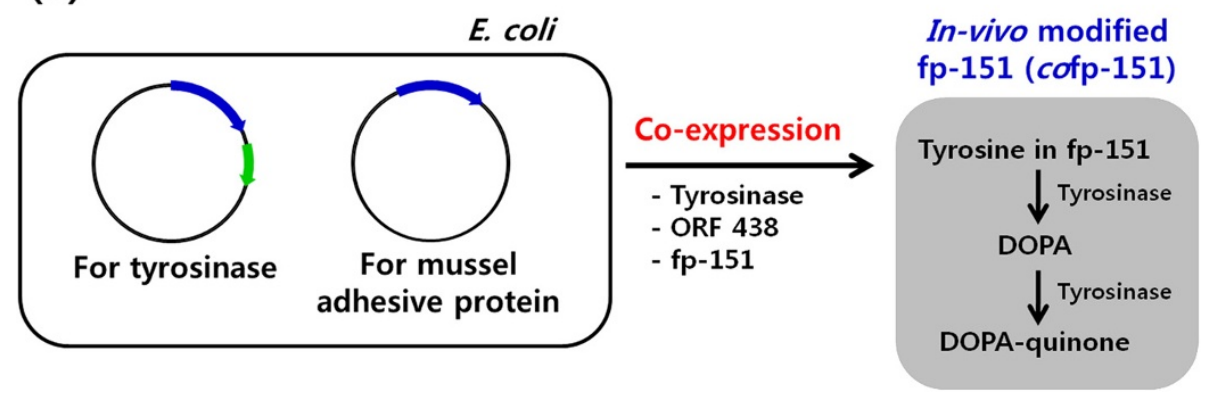

Figure 1 Schematic diagram of the co-expression system to obtain in vivo tyrosine-modified MAP. (A) pACYC-Tyr-438 harboring two genes of tyrosinase and ORF 438 was used for functional expression of tyrosinase (left vector map), and pET-fp151 was used for expression of fp-151 (right vector map). (B) The two recombinant plasmids were introduced into E. coli BL21 (DE3) for co-expression of fp-151, tyrosinase, and ORF 438. Through this co-expression strategy, in vivo tyrosine-modified MAP can be obtained without additional in vitro modification.

sample. However, this fraction was a complex mixture whose main component was cofp-151 (data not shown). The cofp-151 protein might be bound to other components in soluble $E$. coli lysate by its adhesive properties, such as modified tyrosine residues, hydrogen bondforming residues, and the unique conformation of adhesive proteins. Thus, affinity purification was conducted again under denaturing conditions using $8 \mathrm{M}$ urea, and we found that the cofp-151 was able to bind to the resin. Unfortunately, the cofp-151 was eluted poorly under normal elution conditions for hexahistidine $\left(\mathrm{His}_{6}\right)$-tagged proteins, such as $100-250 \mathrm{mM}$ imidazole or weak acidic conditions of $\mathrm{pH}$ 4.5-5.3 (data not shown). Under these conditions, most of the target proteins remained bound to the column resin, but an increased concentration of imidazole and decreased $\mathrm{pH}$ conditions improved the elution yield of the purified protein. Because the purified cofp-151 protein precipitated during the dialysis step to remove the imidazole, the elution was optimized using acidic conditions. Finally, we obtained cofp-151 with above $95 \%$ purity by elution with $0.5 \mathrm{M} \mathrm{HCl}$ (Figure 2B). After dialysis using distilled water, the purified sample was lyophilized and stored at $-80^{\circ} \mathrm{C}$ (Figure 2C).

\section{Analyses of tyrosine modification in cofp-151}

Quinoproteins, which contain quinones and related quinonoid substances such as Dopaquinone and Dopa, are specifically stained by nitroblue tetrazolium (NBT) and glycinate solution because of their redox-cycling, although the intensity is different from quinones and quinonoid compounds [24]. Thus, we first investigated the in vivo modification of tyrosine residues in cofp-151 using the NBT/glycinate staining method. Along with non-modified fp-151 as a negative control and in vitro tyrosine-modified fp-151 ( $m \mathrm{fp}-151)$ as a positive control, in vivo tyrosine-modified cofp-151 was dropped onto a nitrocellulose membrane and stained using NBT/glycinate solution. We found that non-modified fp-151 was not stained at all, but blue-purple spots were clearly shown in the $m f p-151$ and cofp-151 samples (Figure 3A), indicating the tyrosine residues of cofp-151 were successfully in vivo modified into quinones or quinonoid compounds by co-expression of tyrosinase. Importantly, the cofp-151 spot had a higher intensity than the $m f p-151$ spot, indicating the in vivo modification efficiency is higher than the in vitro modification efficiency. Next, we performed liquid-chromatography-mass/mass spectrometry (LC-MS/MS) analysis using a trypsin digest to confirm the presence of quinone relatives in the cofp-151 protein. From the LC-MS/MS analysis, Ala-Lys-Pro-Ser-Dopaquinone-Pro-Pro-Thr-Tyr-Lys fragments were detected, which can be from the repeating decapeptide unit of the fp- 1 region in the hybrid MAP fp-151 (Figure 3B). However, Dopa residues were 


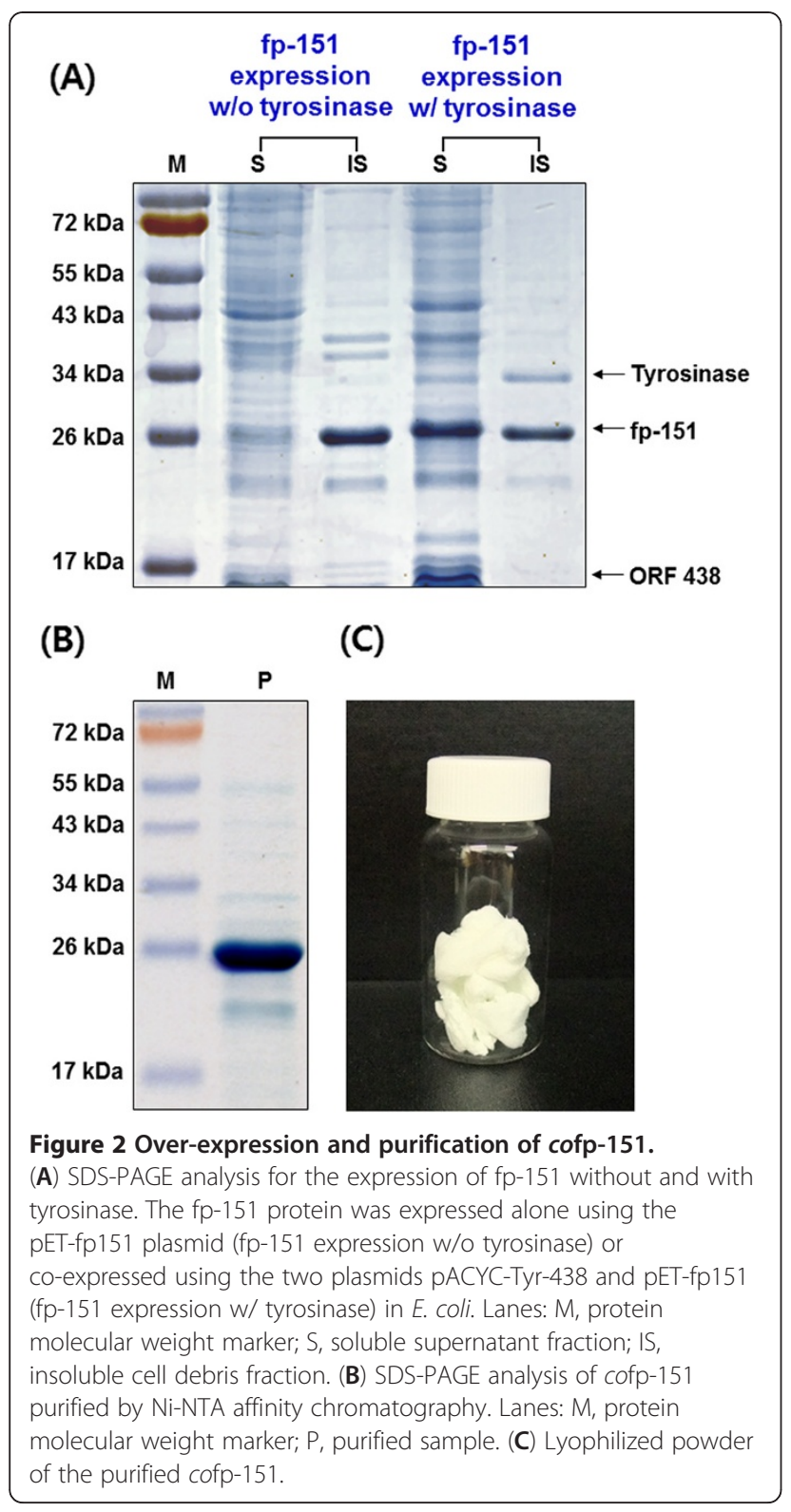

not found in the analysis. These results demonstrated that tyrosines at position 5 in the decapeptide unit of the fp- 1 region in the cofp-151 were in vivo modified into Dopaquinone through co-expression of tyrosinase, although the modification of tyrosine residues was more frequently detected at position 9 than position 5 in the natural fp- 1 [25]. Previously, the tyrosine modification at position 5 was observed in synthetic fp-1 decapeptide conversion using mushroom tyrosinase [9] and was also observed with in vivo modification of fp-151 in insect Sf9 cells [26]. Thus, the altered preference of hydroxylation position might be due to the different modification enzymes and/or the different structural features of fp-151.
In mussel, it is thought that Dopa residues are formed from post-translational modifications catalyzed by polyphenol oxidases [2,27]. Polyphenol oxidases catalyze the $o$-hydroxylation of monophenol to $o$-diphenols (catechols) and also further catalyze the dehydrogenation of $o$-diphenols to $o$-quinones. However, only Dopa has been observed in natural MAPs, and very little information about these enzymes exists. Thus, the conversion mechanism of Dopa molecules from tyrosine residues has yet to be clearly explained. Antioxidant molecules might block Dopa oxidation into Dopaquinone, or undiscovered polyphenol oxidase with high substrate specificity for tyrosine residues (or tyrosine hydroxylase) might be involved in the preparation of Dopa-incorporated MAPs. To the best of our knowledge, polyphenol oxidases have not been identified yet in mussel. Thus far, catechol oxidase activity was measured in the Golgi complex of Mytilus edulis and whole byssus structures $[28,29]$, and nonhomogeneous catechol oxidases were only isolated from the mussel foot and regions of the byssus [30,31].

\section{Bulk adhesive strength of cofp-151}

We expected that the successful in vivo modification of tyrosine residues would demonstrate the superior adhesive properties of MAPs via quinone-derived intermolecular cross-linking. To this end, the bulk-scale adhesive strength of cofp-151 was measured using aluminum adherends due to their ease of use and good surface reproducibility. The adhesive strength of $m \mathrm{fp}$ 151 was also measured to compare the strengths of in vitro and in vivo tyrosine-modified fp-151. Bovine serum albumin (BSA) was used as a negative control. Non-modified fp-151 and lysozyme, which is a basic protein similar to fp-151 [32], were also used as controls. The aluminum adherends were attached under the same conditions using BSA, lysozyme, fp-151, $m \mathrm{fp}-151$, and cofp-151, and the samples were incubated at $37^{\circ} \mathrm{C}$ for 3 $\mathrm{h}$ in air. Under these conditions, the shear strength of cofp-151 $(3.01 \pm 0.62 \mathrm{MPa})$ was 4-fold higher than that of $m f p-151(0.80 \pm 0.20 \mathrm{MPa})$ (Figure 4$)$. The observed shear strength difference of in vivo and in vitro tyrosinemodified fp-151 samples may come from the usage of different kinds of tyrosinase. However, MAPs tend to aggregate in neutral $\mathrm{pH}$ conditions [13-15] and treating higher concentrations of tyrosinase did not improve in vitro modification efficiency (data not shown). It was surmised that the steric hindrance by MAP aggregation could restrict the enzymatic reaction regardless of the types of tyrosinase. Thus, the strategy of in vivo modification through tyrosinase co-expression can overcome the relatively-low-yield problem observed in the in vitro tyrosinase-treated modification. It is important to note that cofp-151 showed significantly strong adhesive 


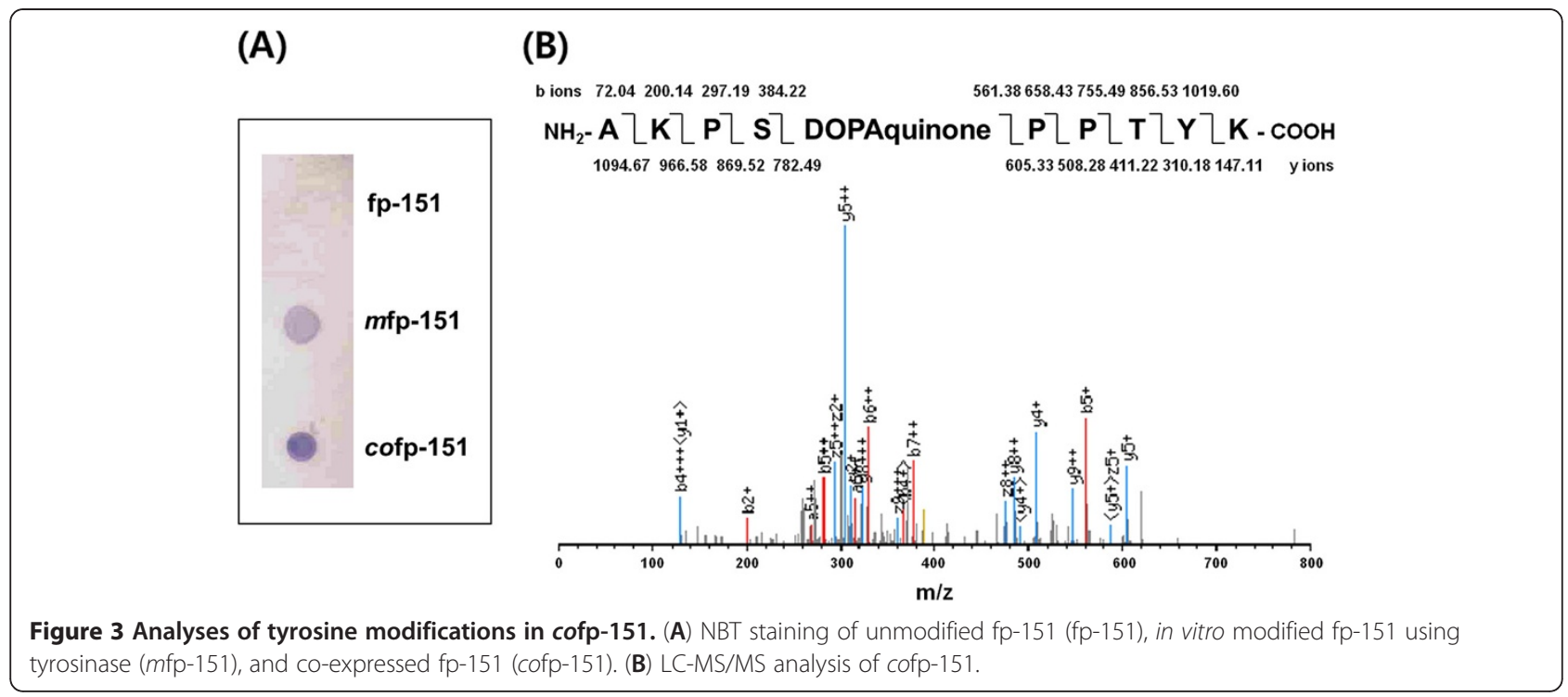

property although the tyrosinase co-expression mainly produced Dopaquinone. It is now accepted that Dopa residues are mainly involved in the remarkable adhesion and the Dopaquinone, which is converted from Dopa, is responsible for the cross-linking $[5,11,33]$. Thus, the quinone-based cross-linking/coupling via radical generation, imine formation, and Michael adduct formation contributes to the strong adhesion [33]. Until now, limited data of the bulk-scale adhesive strengths of MAPs have been reported; overall, adhesive strengths of $0.3 \sim 2$ $\mathrm{MPa}$ were measured for mussel foot extract, synthetic repeats of $\mathrm{fp}-1$ decapeptides, and in vitro tyrosinasetreated recombinant MAPs [12,18,34-38]. The formulation of MAPs through complex coacervation only

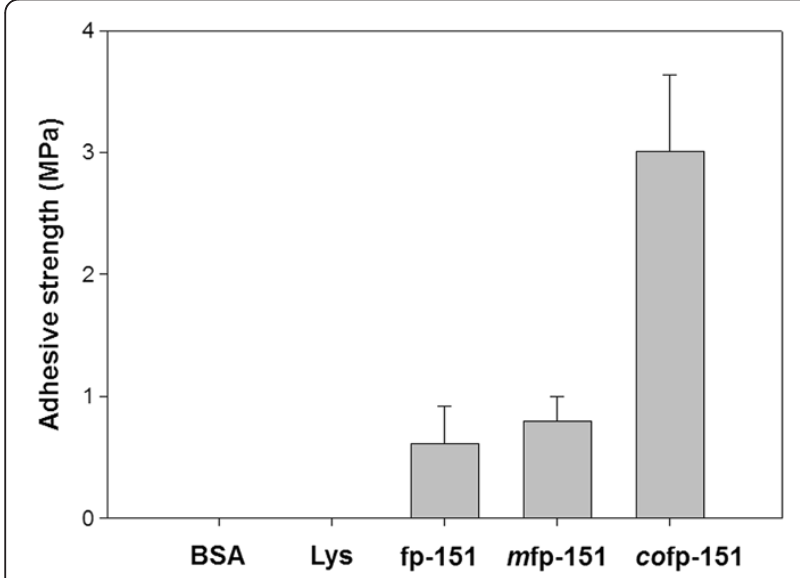

Figure 4 Bulk-scale adhesive strength of cofp-151. BSA, lysozyme (Lys), non-modified fp-151 (fp-151) and mfp-151 were utilized in the control experiments. The aluminum adherends were incubated at $37^{\circ} \mathrm{C}$ for $3 \mathrm{~h}$ in air before the measurements. Each adhesion measurement was repeated at least 5 times and averaged for a given sample. showed adhesive strength above $3 \mathrm{MPa}$ [35]. Thus, the preparation of MAPs using the co-expression strategy for in vivo modification was successful in obtaining MAPs with strong adhesive properties.

\section{Conclusions}

Here, we produced a quinone-incorporated recombinant MAP, fp-151, in E. coli. Quinone residues were successfully incorporated into fp- 151 by the co-expression of fp-151 and tyrosinase, which enhanced the adhesive strength of fp-151 significantly over that of in vitro modified fp-151. This approach can overcome the limitations of conventional in vitro tyrosinase modification of MAPs by directly preparing tyrosine-modified MAPs from E. coli without any additional modification steps after protein purification. Ultimately, we expect that MAPs will be used as bioadhesives with strong adhesive properties and good biocompatibility. The strategy used in this study will be expanded to prepare other Dopa/ Dopaquinone-based adhesive materials including MAPs and accelerate the use of MAPs in practical applications, especially in various biomedical and tissue engineering fields.

\section{Methods}

\section{Strains and vector construction}

E. coli TOP10 (Invitrogen, Carlsbad, CA, USA) was used as a host for recombinant vector preparation and $E$. coli BL21 (DE3) cells (Merck KGaA, Darmstadt, Germany) were used for the co-expression of recombinant fp-151 and both tyrosinase and ORF 438 from S. antibioticus. E. coli cells were grown in Luria-Bertani (LB) medium with $50 \mu \mathrm{g} / \mathrm{mL}$ ampicillin (Sigma, St. Louis, MO, USA). S. antibioticus was obtained from the Korean Collection for Type Culture (DaeJeon, Korea). A single colony of $S$. 
antibioticus was grown in MYG broth $(1 \%(\mathrm{w} / \mathrm{v})$ malt extract, $0.4 \%(\mathrm{w} / \mathrm{v})$ yeast extract, and $0.4 \%(\mathrm{w} / \mathrm{v})$ glucose; $\mathrm{pH} 7.2$ ) at $30^{\circ} \mathrm{C}$. A genomic DNA of S. antibioticus was prepared using genomic DNA purification kit (Promega, Madison, WI, USA). The tyrosinase gene was amplified by polymerase chain reaction (PCR) using two primers of pSA-tyr-5p: 5'-cac caG GAT CCg acc gtc cgc aag aac3' and pSA-tyr-3p: 5'-cac AAG CTT tca gac gtc gaa ggt3'. The amplified product was digested with $B a m \mathrm{HI}$ and HindIII. Similarly, the ORF 438 gene was amplified by PCR using two primers of pSA-438-5p: 5'- cac CAT ATG ccg gaa ctc acc cgt-3' and pSA-438-3p: 5'- cac CTC GAG tca gtt gga ggg gaa-3', and the amplified product was digested using NdeI and XhoI. The digested products were introduced into pACYCDuet-1 plasmid (Merck KGaA, Darmstadt, Germany) using the same restriction enzyme sites. Finally, the introduced DNA sequences were confirmed by sequencing, and the functional tyrosinase expression vector was named pACYC-Tyr-438. In addition, the fp-151 gene was amplified from pENG151 [18] using two primers from the T7 promoter primer: 5'taa tac gac tca cta tag g-3' and p151-NS-His-3pL 5'-cgc AAG CTT gta cgt tgg ag-3'. The amplified product was introduced into the NdeI and HindIII restriction sites of pET-23b (Merck KGaA, Darmstadt, Germany) to remove a stop codon and add a hexahistidine $\left(\mathrm{His}_{6}\right)$ tag for affinity purification (pET-fp151).

\section{Co-expression of $\mathrm{fp}-151$, tyrosinase, and ORF 438}

The constructed recombinant plasmids, pET-fp151 and pACYC-Tyr-438, were introduced into E. coli BL21 (DE3) cells by electroporation for the co-expression of recombinant $\mathrm{fp}-151$, tyrosinase, and ORF 438. A single colony from a freshly streaked plate of the cells was grown in $50 \mathrm{~mL}$ of LB medium with $50 \mu \mathrm{g} / \mathrm{mL}$ ampicillin and $10 \mu \mathrm{g} / \mathrm{mL}$ chloroamphenicol at $37^{\circ} \mathrm{C}$ overnight. 5 $\mathrm{mL}$ of the culture was transferred into $500 \mathrm{~mL}$ of $\mathrm{LB}$ medium with $50 \mu \mathrm{g} / \mathrm{mL}$ ampicillin and $10 \mu \mathrm{g} / \mathrm{mL}$ chloroamphenicol in a $3 \mathrm{~L}$ baffled flask, and cultivated at $37^{\circ} \mathrm{C}$ and $250 \mathrm{rpm}$ until an $\mathrm{OD}_{600}$ of $0.8 \sim 1.0$. Isopropylß-D-thiogalactopyranoside (IPTG; Sigma) (final concentration, $1 \mathrm{mM}$ ) was added for induction of proteins, and cells were further incubated at $37^{\circ} \mathrm{C}$ and $250 \mathrm{rpm}$ for 4 h. As a control, E. coli harboring sole pET-fp151 was also cultured in the same condition. Cells were harvested by centrifugation at $10,000 \mathrm{~g}$ for $10 \mathrm{~min}$ at $4^{\circ} \mathrm{C}$, and the pellets were stored at $-80^{\circ} \mathrm{C}$ for further purification.

The cells were disrupted on ice for $20 \mathrm{~min}$ by sonic dismembrator (Fisher Scientific, Waltham, MA, USA) using a $3 \mathrm{sec}$ pulse on and $7 \mathrm{sec}$ cooling period between each burst. The lysate was centrifuged at 10,000 $g$ for 10 min at $4^{\circ} \mathrm{C}$. The supernatant was collected for soluble fraction analysis. The residual pellet was washed twice with distilled water (DW) and resuspended in the same volume of lysis buffer before the sonication, which was used as insoluble fraction.

\section{Purification of cofp-151}

To purify the co-expressed fp-151 with tyrosinase, the cell pellet was resuspended in $5 \mathrm{~mL}$ of lysis buffer $(50$ $\mathrm{mM}$ sodium phosphate buffer, $300 \mathrm{mM} \mathrm{NaCl}, 10 \mathrm{mM}$ imidazole, and $8 \mathrm{M}$ urea; $\mathrm{pH}$ 7.0) per gram of wet weight. The cells were disrupted on ice for $20 \mathrm{~min}$ by sonic dismembrator (Fisher Scientific) using a $3 \mathrm{sec}$ pulse on and $7 \mathrm{sec}$ cooling period between each burst. The lysate was centrifuged at $10,000 \mathrm{~g}$ for $10 \mathrm{~min}$ at $4^{\circ} \mathrm{C}$. The supernatant was collected and applied to a Ni-NTA resin (Qiagen, Hilden, Germany) for affinity purification. After incubation of the lysate-Ni-NTA mixture for $1 \mathrm{~h}$, the mixture was loaded into a column. The resin was washed three times with 5 column volumes of wash buffer (50 mM sodium phosphate buffer, $300 \mathrm{mM} \mathrm{NaCl}, 30$ $\mathrm{mM}$ imidazole, and $8 \mathrm{M}$ urea; $\mathrm{pH}$ 6.0). Recombinant fp151 protein was eluted using $0.5 \mathrm{M}$ hydrochloric acid. The eluted protein solution was diluted 5 times using DW and dialyzed twice in DW using a dialysis membrane of 12,000 14,000 Da molecular weight cut off (Spectrum Laboratories, Rancho Dominguez, CA, USA). Finally, the purified cofp-151 protein was freeze-dried and stored at $-80^{\circ} \mathrm{C}$. The expression level and purity were monitored by sodium dodecyl sulfatepolyacrylamide gel electrophoresis (SDS-PAGE). Gel-Pro Analyzer $^{\mathrm{TM}}$ (Media Cybermetics Inc., Bethesda, MD, USA) was used to analyze the protein level. The protein concentration was assessed using the Bradford protein assay method (Bio-Rad, Hercules, CA, USA).

\section{NBT staining and LC-MS/MS analysis}

The modifications of tyrosine residues were detected by redox-cycling staining with NBT and glycinate reagent [24]. For the control experiments, $m f$ - 151 was prepared using mushroom tyrosinase (Sigma) based on our previous protocol [35]. Two microliters of $3 \mathrm{mg} / \mathrm{mL} \mathrm{fp}-151$, $m f p-151$, and cofp-151 were dropped onto a nitrocellulose transfer membrane (Protran BA83; Whatman ${ }^{\circledR}, \mathrm{GE}$ healthcare, Little Chalfont, UK). The membrane was immersed in a NBT/glycinate solution $(0.6 \mathrm{mg} / \mathrm{mL} \mathrm{NBT}$ and $2 \mathrm{M}$ potassium glycinate buffer; $\mathrm{pH} 10)$ and incubated at room temperature until a blue-purple color developed. The stained membrane was washed using 0.1 $\mathrm{M}$ sodium borate solution and DW.

For LC-MS/MS analysis, the purified cofp-151 protein $(20 \mu \mathrm{g})$ was dissolved in a digestion solution (6 M urea and $40 \mathrm{mM}$ ammonium bicarbonate dissolved in highperformance LC-grade water). Protein reduction was performed with $5 \mathrm{mM}$ tris(2-carboxyethyl) phosphine hydrochloride for $1 \mathrm{~h}$, followed by an alkylation step 
with $25 \mathrm{mM}$ iodoacetamide in the dark for $30 \mathrm{~min}$ at room temperature. The sample was digested with $5 \mathrm{ng} /$ $\mathrm{mL}$ sequencing-grade modified trypsin (Promega) for 16 $\mathrm{h}$ at $37^{\circ} \mathrm{C}$. All digested samples were collected and desalted with C-18 spin columns (Thermo, Rockford, IL, USA). Tryptic peptides were repeatedly analyzed (5 times) by LC-MS/MS on an LTQ XL mass spectrometer (Thermo Finnigan, San Jose, CA, USA). LC-MS/MS data were analyzed by a computational proteomics analysis system using the $\mathrm{X}$ ! tandem search engine [39] with the database from Uniprot [40].

\section{Measurement of bulk-scale adhesive strength}

Shear strength was analyzed using aluminum adherends (10 $\mathrm{mm}$ in width $\times 100 \mathrm{~mm}$ in length) with a hole placed near the ends [12]. The adherends were etched with $5 \%(\mathrm{w} /$ v) $\mathrm{NaOH}$ solution for $5 \mathrm{~min}$ at room temperature, washed with DW, and immersed in a $\mathrm{HNO}_{3}$ solution $(30 \%(\mathrm{v} / \mathrm{v}))$ for $1 \mathrm{~min}$ to eliminate the smut layer formed by etching. Finally, the adherends were prepared by washing with DW and drying in air at room temperature. To measure the adhesive strength of cofp-151, $10 \mu \mathrm{L}$ of $5 \%$ acetic acid solution was placed at the end of each adherend. When $5 \mathrm{mg}$ of the lyophilized cofp-151 powder was added to one solution drop, the powder was quickly dissolved in the solution which produced a highly viscous emulsion. The viscous solution was mixed and spread well on the adhesion area $(10 \mathrm{~mm} \times 10 \mathrm{~mm})$ of an adherend with a spatula. Then, another adherend was placed on the cofp-151 solution-coated surface with the $10 \mathrm{~mm} \times 10 \mathrm{~mm}$ overlap area. The samples were incubated at $37^{\circ} \mathrm{C}$ for $3 \mathrm{~h}$ in air before measurements. The shear strength was directly obtained with a universal material testing machine (Instron 3344; Instron, Norwood, MA, USA) with a $2000 \mathrm{~N}$ load cell. These adhesion studies were an adaptation of the ASTM D1002 standard method (ASTM International D1002-05, 2005). Each adhesion measurement was repeated at least 5 times and averaged for a given sample.

BSA (Sigma), lysozyme (Sigma), non-modified fp-151, and $m f \mathrm{f}-151$ were used at $500 \mathrm{mg} / \mathrm{mL}$ for the control experiments. $m \mathrm{fp}-151$ was prepared by in vitro modification of fp-151. fp-151 protein $(2 \mathrm{mg} / \mathrm{mL})$ was incubated overnight at $37^{\circ} \mathrm{C}$ in phosphate-buffered saline (PBS; $2.68 \mathrm{mM} \mathrm{KCl}, 13.7 \mathrm{mM} \mathrm{NaCl}, 1.47 \mathrm{mM} \mathrm{KH}_{2} \mathrm{PO}_{4}$, and $0.875 \mathrm{mM} \mathrm{Na}_{2} \mathrm{HPO}_{4}$ ) with $25 \mathrm{mM}$ ascorbic acid as a reducing agent and with $50 \mu \mathrm{g} / \mathrm{mL}$ mushroom tyrosinase (Sigma). Then, the resultant $m \mathrm{fp}-151$ was freezed-dried after the dialysis twice against DW.

\section{Competing interests}

The authors declare that they have no competing interests.

\section{Author's contributions}

YSC and HJC designed research. YSC, YJY and BY performed the experiments and analyzed the results. YSC and HJC wrote the paper. All authors have read and approved final version of the manuscript.

\section{Acknowledgements}

This work was supported by the Marine Biotechnology Program funded by the Ministry of Land, Transport and Maritime Affairs of Korea and the Technology Innovation Program (\#10041466) funded by the Ministry of Knowledge Economy of Korea. We thank Dr. Daehee Hwang (POSTECH) for his LC-MS/MS analysis.

\section{Author details}

'Department of Chemical Engineering, Chungnam National University, Daejon 305-764, Korea. ${ }^{2}$ Department of Chemical Engineering, Pohang University of Science and Technology, Pohang 790-784, Korea. ${ }^{3}$ Ocean Science and Technology Institute, Pohang University of Science and Technology, Pohang 790-784, Korea.

Received: 12 July 2012 Accepted: 21 October 2012 Published: 24 October 2012

\section{References}

1. Kamino K: Underwater adhesive of marine organisms as the vital link between biological science and material science. Mar Biotechnol (NY) 2008, 10:111-121.

2. Silverman $\mathrm{HG}$, Roberto FF: Understanding marine mussel adhesion. Mar Biotechnol (NY) 2007, 9:661-681.

3. Hammer DA, Tirrell M: Biological adhesion at interfaces. Annu Rev Mater Sci 1996, 26:651-691.

4. Waite $\mathrm{JH}$ : Precursors of quinone tanning: dopa-containing proteins. Methods Enzymol 1995, 258:1-20.

5. Sagert J, Sun C, Waite JH: Chemical subtleties of mussel and polychaete holdfasts. In Biological adhesives. Edited by Smith AM, Callow JA. Berlin: Springer-Verlag; 2006:125-140.

6. Cha HJ, Hwang DS, Lim S: Development of bioadhesives from marine mussels. Biotechnol J 2008, 3:631-638.

7. Zhao H, Sun C, Stewart RJ, Waite $\mathrm{JH}$ : Cement proteins of the tube-building polychaete Phragmatopoma californica. J Biol Chem 2005, 280:42938-42944.

8. Strausberg RL, Link RP: Protein-based medical adhesives. Trends Biotechnol 1990, 8:53-57.

9. Marumo K, Waite $\mathrm{JH}$ : Optimization of hydroxylation of tyrosine and tyrosine-containing peptides by mushroom tyrosinase. Biochim Biophys Acta 1986, 872:98-103.

10. Yu ME, Hwang JY, Deming TJ: Role of L-3,4-dihydroxyphenylalanine in mussel adhesive proteins. J Am Chem Soc 1999, 121:5825-5826.

11. Lee $H$, Scherer NF, Messersmith PB: Single-molecule mechanics of mussel adhesion. Proc Natl Acad Sci U S A 2006, 103:12999-13003.

12. Cha HJ, Hwang DS, Lim S, White JD, Matos-Perez CR, Wilker JJ: Bulk adhesive strength of recombinant hybrid mussel adhesive protein. Biofouling 2009, 25:99-107.

13. Haemers S, Koper GJ, Frens G: Effect of oxidation rate on cross-linking of mussel adhesive proteins. Biomacromolecules 2003, 4:632-640.

14. Haemers S, van der Leeden MC, Koper GM, Frens G: Cross-linking and multilayer adsorption of mussel adhesive proteins. Langmuir 2002 18:4903-4907.

15. Lim S, Choi YS, Song YH, Cha HJ: Salt effects on aggregation and adsoprtion characteristics of recombinant mussel adhesive protein $\mathrm{fp}$ 151. J Adhesion 2009, 85:812-824.

16. Taylor SW: Chemoenzymatic synthesis of peptidyl 3,4dihydroxyphenylalanine for structure-activity relationships in marine invertebrate polypeptides. Anal Biochem 2002, 302:70-74.

17. Jee JG, Park SJ, Kim HJ: Tyrosinase-induced cross-linking of tyrosinecontaining peptides investigated by matrix-assisted laser desorption/ ionization time-of-flight mass spectrometry. Rapid Commun Mass Spectrom 2000, 14:1563-1567.

18. Hwang DS, Gim Y, Yoo HJ, Cha HJ: Practical recombinant hybrid mussel bioadhesive fp-151. Biomaterials 2007, 28:3560-3568.

19. Selzer G, Som T, Itoh T, Tomizawa J: The origin of replication of plasmid p15A and comparative studies on the nucleotide sequences around the origin of related plasmids. Cell 1983, 32:119-129.

20. Della-Cioppa G, Garger SJ, Sverlow GG, Turpen TH, Grill LK: Melanin production in Escherichia coli from a cloned tyrosinase gene. Biotechnology (NY) 1990, 8:634-638.

21. Claus H, Decker H: Bacterial tyrosinases. Syst Appl Microbiol 2006, 29:3-14. 
22. Hwang DS, Gim Y, Cha HJ: Expression of functional recombinant mussel adhesive protein type 3A in Escherichia coli. Biotechnol Progr 2005, 21:965-970.

23. Hwang DS, Yoo HJ, Jun JH, Moon WK, Cha HJ: Expression of functional recombinant mussel adhesive protein Mgfp-5 in Escherichia coli. Appl Environ Microbiol 2004, 70:3352-3359.

24. Paz MA, Fluckiger R, Boak A, Kagan HM, Gallop PM: Specific detection of quinoproteins by redox-cycling staining. J Biol Chem 1991, 266:689-692.

25. Waite $\mathrm{JH}$, Housley $\mathrm{TJ}$, Tanzer ML: Peptide repeats in a mussel glue protein: theme and variations. Biochem 1985, 24:5010-5014.

26. Lim S, Kim KR, Choi YS, Kim D-K, Hwang D, Cha HJ: In vivo posttranslational modifications of recombinant mussel adhesive protein in insect cells. Biotechnol Prog 2011, 27:1390-1396.

27. Waite $\mathrm{JH}$ : The phylogeny and chemical diversity of quinone-tanned glues and varnishes. Comp Biochem Physiol B 1990, 97:19-29.

28. Waite JH: Catechol oxidase in the byssus of the common mussel, Mytilus edulis L. J Mar Biol Assoc UK 1985, 65:359-371.

29. Zuccarello LV: Ultrastructural and cytochemical study on the enzyme gland of the foot of a mollusc. Tissue Cell 1981, 13:701-713.

30. Burzio VA, Silva T, Pardo J, Burzio LO: Mussel adhesive enhances the immobilization of human chorionic gonadotrophin to a solid support. Anal Biochem 1996, 241:190-194.

31. Hellio C, Bourgougnon N, Gal YL: Phenoloxidase(E.C. 1.14.18.1) from the byssus gland of Mytilus edulis: purification, partial characterization, and application for screening products with potential antifouling activities. Biofouling 2000, 16:235-244.

32. Tanford C, Wagner ML: Hydrogen ion equilibria of lysozyme. J Am Chem Soc 1954, 76:3331-3336.

33. Deming TJ: Mussel byssus and biomolecular materials. Curr Opin Chem Biol 1999, 3:100-105.

34. Choi YS, Kang DG, Lim S, Yang YJ, Kim CS, Cha HJ: Recombinant mussel adhesive protein fp-5 (MAP fp-5) as a bulk bioadhesive and surface coating material. Biofouling 2011, 27:729-737.

35. Lim S, Choi YS, Kang DG, Song YH, Cha HJ: The adhesive properties of coacervated recombinant hybrid mussel adhesive proteins. Biomaterials 2010, 31:3715-3722.

36. Gim Y, Hwang DS, Lim S, Song YH, Cha HJ: Production of fusion mussel adhesive fp-353 in Escherichia coli. Biotechnol Progr 2008, 24:1272-1277.

37. Ninan L, Monahan J, Stroshine RL, Wilker JJ, Shi R: Adhesive strength of marine mussel extracts on porcine skin. Biomaterials 2003, 24:4091-4099.

38. Yamamoto H: Synthesis and adhesive studies of marine polypeptides. J Chem Soc Perkin Trans 1987, 1:613-618.

39. Bjornson RD, Carriero NJ, Colangelo C, Shifman M, Cheung KH, Miller PL, Williams K: X!!Tandem, an improved method for running X!tandem in parallel on collections of commodity computers. J Proteome Res 2008, 7:293-299.

40. Wu CH, Apweiler R, Bairoch A, Natale DA, Barker WC, Boeckmann B, Ferro S, Gasteiger E, Huang H, Lopez R, Magrane M, Martin MJ, Mazumder R, O'Donovan C, Redaschi N, Suzek B: Nucleic Acids Res 2006, 34(database issue):D187-D191.

doi:10.1186/1475-2859-11-139

Cite this article as: Choi et al:: In vivo modification of tyrosine residues in recombinant mussel adhesive protein by tyrosinase co-expression in Escherichia coli. Microbial Cell Factories 2012 11:139.

\section{Submit your next manuscript to BioMed Central and take full advantage of:}

- Convenient online submission

- Thorough peer review

- No space constraints or color figure charges

- Immediate publication on acceptance

- Inclusion in PubMed, CAS, Scopus and Google Scholar

- Research which is freely available for redistribution

Submit your manuscript at www.biomedcentral.com/submit
C Biomed Central 